\title{
Gender/sex, race/ethnicity, similarities/differences among SARS-CoV, MERS-CoV, and COVID-19 patients
}

\author{
Freedom L Ha BS, Albin John BA, MBA, Mimi Zumwalt MD
}

\begin{abstract}
SARS-CoV-2 is a novel virus that has resulted in a global pandemic since the first cases were reported in late 2019 from Wuhan, China. It is also a coronavirus that shares similarities to SARS-CoV and MERS-CoV with respect to pathophysiology, transmission, and affected populations. A review of the literature was conducted to explain possible underlying reasons for the difference in coronaviruses' effects on certain demographics with a focus on gender/ sex and race/ethnicity. Both male and female genders vary in their biological makeup, including immunity, hormones, and physiological components, such as angiotensin-converting enzyme 2 (ACE2). These variations seem to give moderate support to the data that demonstrate a higher mortality rate of COVID-19 in males. Yet, these factors must be investigated further since the current studies are limited in scope and sample size as the COVID-19 pandemic continues to evolve. Information involving COVID-19 racial and ethnic data currently is limited, as most countries have not recorded cases based on race or ethnicity, but primarily age and gender. The United States Centers for Disease Control and Prevention (CDC) limited data have shown that racial minorities, such as African-Americans, are more likely to have worse outcomes. This could be due to other parameters, including healthcare disparities, biological factors, and socioeconomic status. Further research in understanding both gender/sex and race/ethnicity with respect to respiratory viruses, including COVID-19, could potentially improve the poor prognosis seen among particular demographics.
\end{abstract}

Keywords: SARS-CoV-2, COVID-19, MERS-CoV, Middle Eastern Respiratory Syndrome, SARS, SARS-CoV, Severe Acute Respiratory Syndrome, ethnicity, race, minority, gender, sex

\section{INTRODUCTION}

The emergence of a novel virus shook the world in December 2019 when reports began to surface from Wuhan, China, of patients displaying symptoms of acute respiratory syndrome. The disease was thought to have spread from a local market in Wuhan with associated symptoms including fever, dry cough, dyspnea, pneumonia, and headache that could

Corresponding author: Mimi Zumwalt

Contact Information: Mimi.Zumwalt@ttuhsc.edu

DOI: 10.12746/swrccc.v9i37.795 progress to respiratory failure. ${ }^{1}$ Based on the sequencing of its genome from early patient samples, this new virus shared $79 \%$ similarity to the SARS-CoV virus from 2003 , and $50 \%$ similarity to the MERS-CoV virus from 2012. ${ }^{2}$ Therefore, the virus was placed within the Coronaviridae family and named SARS-CoV-2 or COVID-19, as it has become more commonly known. This emerging viral infection was declared a pandemic by the WHO on March 11, 2020, ${ }^{3}$ and as of May 6, 2020, there are 3,595,662 cases of COVID-19 infection worldwide. ${ }^{4}$

The Coronaviridae family includes seven coronaviruses that can infect humans: four (HKU1, NL63, OC43, and 229E) cause mild symptoms, and three 


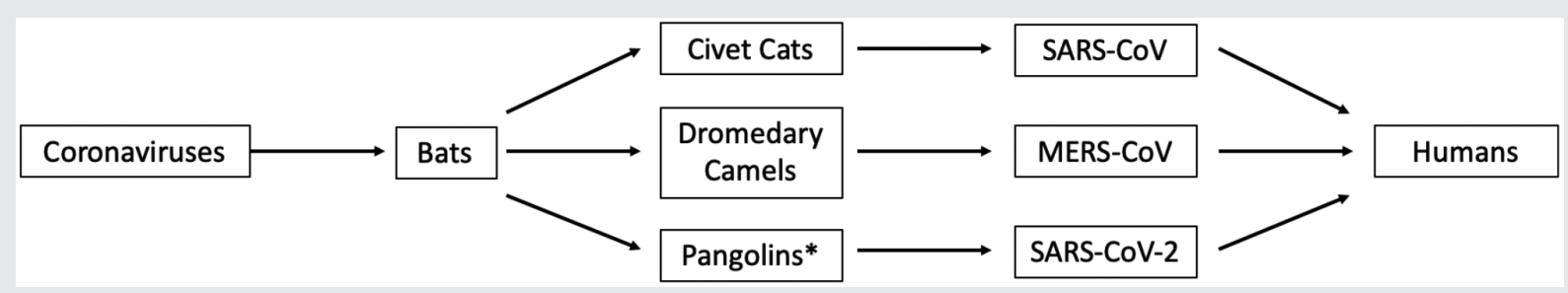

Figure 1. Transmission of coronaviruses adapted from Firas et al. ${ }^{6}$

cause severe respiratory illnesses that include SARSCoV, MERS-CoV, and now COVID-19. ${ }^{5}$ All three of these viruses' mechanism of entry involves spreading from person to person by contact with origination from bats contracting such coronaviruses. ${ }^{6}$ Prior pathogens SARS-CoV and MERS-CoV were transmitted from bats to civet cats and dromedary camels, respectively, while the animal of transmission for SARS-CoV-2 has been linked to a pangolin due to their genetic similarities (Figure 1). ${ }^{7}$

The SARS-CoV outbreak that began in Asia in February 2003 presented with symptoms of fever, headache, body aches, dry cough, and pneumonia. This disease resulted in 8,098 cases worldwide with 774 deaths. $^{8}$ The MERS-CoV outbreak emerged in Saudi Arabia in 2012 with similar symptoms, but most patients also developed severe respiratory illness. ${ }^{9}$ There were 2,494 total cases of MERS-CoV with 858 deaths resulting in a mortality rate of $37 \%$, much higher than SARS-CoV at $10 \% .{ }^{10}$ The worldwide mortality rate of COVID-19 varies greatly between countries; in Belgium the death rate is as high as $15.9 \%$, whereas in Armenia the death rate is $1.5 \%{ }^{11}$ The mortality rate of SARS-CoV-2 will likely not be accurate until the spread begins to slow down. While the mortality rate of COVID-19 is lower than MERS-CoV and in the same range as SARS-CoV, the global case count is astronomically higher with over 3 million cases and 247,652 deaths as of May 6, 2020. ${ }^{4}$ With these alarming statistics, there is a drastic need to explore the underlying factors that contribute to the immense spread of this virus. Unfortunately, COVID-19 has spared no particular group; it has infected and killed the young, the old, males, and females in practically every country across the world. Statistically, there are variations in whether males or females comprise the majority of cases dependent on the region, but it is known that the majority of deaths are male. ${ }^{12}$

In Europe as of April 26, 2020, available case data from the World Health Organization showed $61 \%$ of COVID-19 deaths have been in males with $95 \%$ having at least one underlying condition, such as diabetes or cardiovascular disease..$^{13}$ These data also indicated that males make up the majority of hospitalizations and ICU admissions. In the United States (US) as of April 25, 2020, 52.8\% of laboratory-confirmed COVID19 cases are males; females make up $47.2 \% .{ }^{14}$ In the United States, African-Americans make up $13.4 \%$ of the population, Hispanic or Latino $18.3 \%$, Asian $5.9 \%$, and White $76.5 \% .{ }^{15}$ Yet, according to the CDC as of April 25, 2020, African-Americans make up $36.4 \%$ of laboratory-confirmed COVID-19 cases, while Whites consist of $40.2 \% .{ }^{14}$ With respect to race and ethnicity, the CDC have reported that minorities, especially African-Americans, have had disproportionate death rates from COVID-19 infection. ${ }^{16}$

A systematic literature review was performed to analyze possible underlying reasons for the differences in disease presentation, severity, and outcomes observed in COVID-19 patients. The results of the following data search will be discussed with emphasis on the differences in various biological, clinical, and social differences seen with regard to gender/sex and race/ ethnicity.

\section{Methods}

The following databases/search engines were used to search for scientific reviews, analyses, case studies, and primary research: Google Scholar, PubMed, and ScienceDirect. The date restrictions were placed 
between 2000-2020, with a preference to select more recent papers within the same topic scope. The primary keywords used to search for information included: SARS-CoV-2, COVID-19, MERS-CoV, MERS, Middle Eastern Respiratory Syndrome, SARS, SARS-CoV, Severe Acute Respiratory Syndrome, race, ethnicity, minority, gender, and sex.

The authors conducted their respective searches on separate topics to avoid collecting redundant papers. The last search was completed on May 6, 2020. The references listed for each paper were also reviewed for any information that could lend clarity to the topic. Of the selected studies, the most relevant results and figures were included in this article to promote understanding and support the overall topic of the review. Only studies written in English were used. The sources were evaluated using the following criteria:

1. Provided information relevant to gender/sex and/or race/ethnicity in the context of coronaviruses.

2. Explained a mechanism or physiological phenomena that proved to guide understanding of the coronaviruses.

3. Published or in the process of publication through a peer-reviewed journal or a reputable government agency.

\section{IMMUNITY AND HORMONES IN GENDER/SEX}

A human's sex is defined by the presence of sex chromosomes, reproductive organs, and sex steroid production. Sex contributes to the anatomical and physiological mechanisms in which a human can contract and clear infectious diseases, such as viruses. Gender is a broader term that encompasses societal norms regarding masculinity and femininity. Both of these characteristics contribute to how one's body responds to a pathogen, and the terms are used interchangeably in the literature. The defined biological sexes in humans are male and female, with males having an $X$ and $Y$ chromosome, while females have two $X$ chromosomes. ${ }^{17}$

The $\mathrm{X}$ chromosome encodes immune-related genes for both adaptive and innate immunity. $\mathrm{X}$ chromosome inactivation in females allows some genes to be modulated to promote a stronger immune response as compared to males who have no "backup" $X$ chromosome. ${ }^{18}$ Given this fact, females are more likely to develop autoimmune pathology while males tend to be more susceptible to infectious agents. Innate immunity can be thought of as the first line of defense to invading pathogens, including macrophages, toll-like receptors, neutrophils, natural killer cells, the complement system, and dendritic cells. Adaptive immunity involves the latent, long term defense adaptation of the body to pathogens, conferring a "memory" that promotes a more vigorous immune response with subsequent biologically similar pathogens. The main components of the system are $T$ cells and $B$ cells. $B$ cells are responsible for the production of antibodies that neutralize pathogens via interaction with other cells such as macrophages. Generally, despite age differences, females have higher basal immunoglobulin levels, a greater number of $B$ cells, and greater antibody responses than males. ${ }^{17}$

The prevalence and intensity of viral infections tend to be more marked in males, but when females are infected, the disease outcome tends to be worse. The robust immune response of females to infectious virus particles most likely contributes to such adverse reactions during clinical management and development of inflammatory responses. The benefits of prophylactic treatment and therapy for viruses can differ between each gender, dependent on the viral disease. Therefore, the final outcomes in the treatment and response to clinical therapy of viral disease can vary between males and females. ${ }^{19}$

A comparison study in March 2020 by Zeng et al. investigated the serum IgG antibody levels of 331 COVID-19 positive patients (127 males/204 females). They looked for significant differences in levels between sexes that could explain the greater mortality rates and symptom severity seen in males. The 331 patients were divided into four categories by symptomatic presentation: mild (22), general (87), severe (22), and recovering (200). The percentage of males and females and their average ages are reported in Table 1. Of note, the severe group had a higher average age range than the other groups. The levels of $\lg G$ within each group were then analyzed. Female IgG 
Table 1. Data from Zeng et al. ${ }^{20}$

\begin{tabular}{|l|c|c|c|}
\hline Disease Severity & Males (M) & Females (F) & Average Age (M/F) \\
\hline Mild $(\mathrm{n}=22)$ & $36.4 \%(8)$ & $63.6 \%(14)$ & $45.2 / 42.2$ \\
\hline General $(\mathrm{n}=87)$ & $42.5 \%(37)$ & $57.5 \%(50)$ & $46.2 / 49.4$ \\
\hline Severe $(\mathrm{n}=22)$ & $50 \%(11)$ & $50 \%(11)$ & $59.4 / 63.1$ \\
\hline Recovering $(\mathrm{n}=200)$ & $35.5 \%(71)$ & $64.5 \%(129)$ & $49.6 / 50.1$ \\
\hline Total $(\mathrm{n}=331)$ & 127 & 204 & - \\
\hline
\end{tabular}

levels increased in progression from mild to severe, then decreased in recovering patients; male levels only increased from mild to general before decreasing in severe and recovering groups. In the severe group, most female patients had antibody levels greater than $100 \mathrm{AU} / \mathrm{mL}$, while males were lower than $100 \mathrm{AU} / \mathrm{mL}$. Therefore, within the severe group, females tended to have higher IgG levels than males. ${ }^{20}$

Next, the proportion of SARS-CoV-2 IgG antibody levels within set ranges $(0-10 \mathrm{AU} / \mathrm{mL}, 10-100 \mathrm{AU} / \mathrm{mL}$, $100-150 \mathrm{AU} / \mathrm{mL}$, and $150-200 \mathrm{AU} / \mathrm{mL}$ ) of the severe cases was analyzed (Figure 2). While the range of 0-10 AU/mL was similar between the sexes, the ranges of $10-100 \mathrm{AU} / \mathrm{mL}$ and $150-200 \mathrm{AU} / \mathrm{mL}$ had large differences. IgG levels for $63.6 \%$ males were in the range of $10-100 \mathrm{AU} / \mathrm{mL}$, while only $27.27 \%$ of female levels were in this range. In contrast, at the higher range of $150-200 \mathrm{AU} / \mathrm{mL}$, female levels constituted $54.55 \%$, with males at $9.09 \%$. The severe group was then divided into a high $(100-200 \mathrm{AU} / \mathrm{mL})$ and low $(0-100 \mathrm{AU} / \mathrm{mL})$ subgroup, with statistical analysis showing no difference between the low-level group, while the high-level group showed females to be higher than males. Levels of IgG antibody were also analyzed after disease onset that showed females to have a higher level than males at 2-4 weeks post onset but was not apparent after 4 weeks. ${ }^{20}$

This study demonstrated that females produce higher IgG levels in severe COVID-19 cases than males and have a more robust IgG production in early disease stages of this viral infection. Since females usually generate a more robust immune response, these results may help explain the differences in mortality rates of males and females. The limitations of the study included a small sample size from the same hospital, no patients in critical condition were analyzed, and the rate of severe cases for the sexes did not show a consistent trend. The study was funded by the
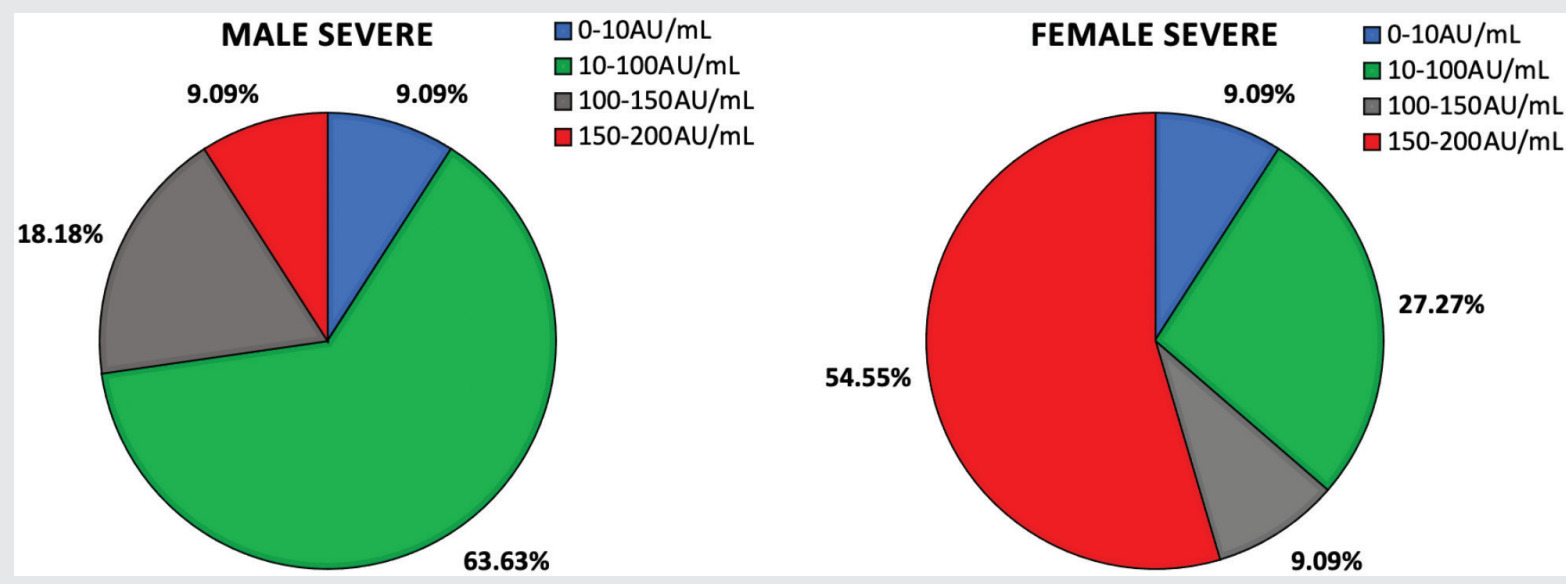

Figure 2. Data from Zeng et al. ${ }^{20}$ 
Department of Clinical Laboratory at Union Hospital of Tongji Medical College, Huazong University of Science and Technology based in Wuhan, Hubei, China. ${ }^{20}$

The differences in immunity between the sexes likely contribute to the differences in infectious manifestation, but hormonal differences also have a role. Hormones bind to intracellular receptors that modulate gene expression, including transcription and translation. The principal hormones produced by males are androgens; females produce estrogens and progesterone. Sex hormone levels change over the course of the human lifespan with the initiation of production starting at puberty and progressively decreasing in older age..$^{21}$

Sex hormones have been shown to directly attach onto immune related gene promoter regions or bind other transcription factors, such as NF-kB (nuclear factor- $\mathrm{KB}$ ), that control the inflammatory response. This modulation indicates the major role that sex hormones have in innate immunity and inflammation regulation. ${ }^{22}$ Some immune cells contain varying amounts of sex hormone receptors, with females showing a more prevalent quantity of their hormone receptors on innate immune cells than males (Table 2) ${ }^{21}$ Generally, testosterone tends to attenuate the innate immune response, while estrogens can stimulate or suppress it. These variances between the sex hormones' effects on immunity seen in the literature do not give a definitive picture. Regardless, infant females have shown to have superior innate immunity compared to male infants, indicating that not only hormones but sex chromosomes also comprise an independent and coexisting factor in immunological sex differences. Although the underlying mechanisms of both sex hormones and chromosomes are not clearly explained, the varying responses to infection between genders do give some weight to their contributions. ${ }^{22}$

A murine model study conducted by Channappanavar et al. investigated sex-based disease outcomes in SARS-CoV by infecting male/female mice of different ages with SARS-CoV to determine susceptibility to infection. Various aged mice of both sexes were used: 8-9 weeks, 5 months, 8-10 months, and 18-20 months. They were infected with SARS-CoV (MA15) and monitored. Antibody/flow cytometry, mRNA quantitative PCR, immunohistochemistry, ELISA, and hormone blockers were all used for evaluation. ${ }^{23}$

Male and female mice were given different amounts of SARS-CoV dosage (MA15). Using 5000 plaque-forming units (PFU) of MA15 in the 8-10-month age group resulted in $90 \%$ mortality in males and $20 \%$ mortality in females. At this dose, young 8-week-old mice of both sexes showed complete resistance, while in the 8-9 month rats all males died, but only $10 \%$ of females died. In the 20-month-old group, male mice

Table 2. Data Adapted from Kadel et al. ${ }^{21}$

\begin{tabular}{|l|c|c|c|c|}
\hline \multicolumn{5}{|c|}{ Sex Steroid Receptors in Human Cells } \\
\hline Cell Type & ER $\alpha$ & ER $\beta$ & PRs & AR \\
\hline Natural killer cells & Yes & Yes & Yes & - \\
\hline Gamma delta T cells & & & Yes & \\
\hline Neutrophils & Yes & Yes & No & Yes \\
\hline Eosiniphils & $-/+$ & $-/+$ & No & No \\
\hline Plasmacytoid dendritic cells & Yes & Yes & - & - \\
\hline Monocytes & Yes & Yes & - & - \\
\hline Dendritic cell & Yes & Yes & - & - \\
\hline Macrophage & Yes & Yes & Yes & Yes \\
\hline Hematopoietic stem cell & Yes & Yes & - & Yes \\
\hline
\end{tabular}

"Yes" or "No" indicates the presence of a sex steroid receptor in a certain cell type based on scientific literature.

,$-<50$ counts; $-/+, 50-100$ counts;,$+ 100-300$ counts; + or - is dependent on receptors in a certain tissue. 
began to die at 1000 PFU of dosing and female mice lived; all 20-month-old mice died at 5000 PFU. When MA15 was increased to 10,000 PFU, all males died, and $40 \%$ of females lived. MA15 viral titers, lung vascular leakage, alveolar edema, and terminal bronchiolar epithelial sloughing were all higher in male mice compared to females. Cells from 9-month-old mice demonstrated increased inflammatory monocytemacrophages (IMMs) and neutrophils in the male lung tissue. Male mice had higher cytokine mRNA levels (IL6, CXCL1, and CCL2) in their lungs than female mice at day 3 post infection; IFN- $\beta$ levels were similar. In addition, greater numbers of IMMs in male mice expressed inflammatory cytokines than IMMs in female mice. Depleting IMMs in female mice showed no change in mortality, but depleting IMMs in male mice had a protective effect, suggesting that inflammatory responses could explain higher male mortality rates. ${ }^{23}$

When T/B cell deficient 8-9-month-old mice were infected with MA15, mortality was higher in male mice, indicating $T$ cell and antibodies are likely not a contributor in the sex differences. Blocking androgens in male mice with a gonadectomy or anti-androgen administration did not change mortality after MA15 infection. Yet SARS-CoV infection itself has been shown to reduce testosterone levels. On the other hand, blocking estrogen production in female mice via ovariectomy increased their mortality to $85 \%$, and estrogen receptor blockers made females more susceptible to MA15 infection compared to an estrogen receptor agonist. The ovariectomized female mice lungs had increased IMMs, but not neutrophils, consistent with the fact that female hormones are known to downregulate the inflammatory response. With the increased IMMs seen in estrogen deprived females, this suggests that estrogen binding receptors promote an anti-inflammatory response in the lungs. Estrogen binding to its receptor showed a protective effect, while androgens did not change mortality rates. Overall, the same phenomenon seen in the sex bias of SARS-CoV infection in humans was demonstrated in this mouse study. ${ }^{23}$

Karlberg et al. analyzed SARS-CoV patient data from the Hong Kong Department of Health from March 2003 to September 22, 2003, with regard to sex, age, and disease history. Data were collected on 1755 patients from the 2003 SARS outbreak in Hong Kong with a case fatality rate of $17 \%$ as of September 22, 2003 (170 males, 129 females). The case fatality of males was $21.9 \%$ $(p<0.0001)$ compared to females at $13.2 \%$, with healthcare workers excluded. The male relative mortality risk estimate was 1.66 (95 percent Cl: 1.35, 2.05) compared to females, and 1.62 (95 percent $\mathrm{Cl}: 1.21,2.16)$ after factoring in age. The females were much younger than males, likely due to hospital staff such as nurses who were infected (22\%) among the 1755 patients. Yet, this group likely did not show a bias in gender differences since most of the deaths occurred over the age of 44 , while most infected health care workers were females under the age of 45 years old. The SARS-CoV mortality gender difference decreased from younger to older patients, perhaps due to the health deterioration associated with increasing age. Hence, males seemed to be more severely affected by SARS-CoV than females, yet the underlying mechanism is still unclear. ${ }^{24}$

Jansen et al. analyzed data from MERS-CoV cases in the Republic of Korea, which had the largest outbreak outside Saudi Arabia. ${ }^{25}$ Data were collected on 183 MERS-CoV cases from May 20 to June 30, 2015 (110 males and 73 females). The results indicated a sex bias toward men in susceptibility to MERS-CoV, which echoed the same trend seen in MERS-CoV studies of the Middle East. While males were more susceptible, the researchers demonstrated that females were more exposed to the virus. This was related to the prevalence of female health care workers (at least three times more than males). These investigators noted lifestyle factors, such as smoking, aggravated the infectious disease. Similar life habits were specifically higher involving males than females in the Republic of Korea. Although a limitation of this study was the small cohort, these results demonstrate the relevance of accounting for sex bias when assessing viral outbreaks such as MERS-CoV. ${ }^{25}$

A case series from the Department of Respiratory and Critical Care Medicine at Beijin Tongren Hospital by Jin et al. compared gender differences in severity and mortality in SARS-CoV-2 and SARS-CoV patients. Data were collected of the following: 43 COVID-19 positive patients treated at this hospital from January 29, 2020 to February 15, 2020, the first 37 COVID-19 
Table 3. Data Adapted from Jin et al. ${ }^{26}$

\begin{tabular}{|l|c|c|c|c|}
\hline \multicolumn{5}{|c|}{ Patients with COVID-19 and SARS-CoV } \\
\hline & \multicolumn{2}{|c|}{ COVID-19 } & \multicolumn{2}{c|}{ SARS-CoV } \\
\hline & $\begin{array}{c}\text { Deceased } \\
(\mathbf{n}=\mathbf{3 7})\end{array}$ & $\begin{array}{c}\text { Survived } \\
(\mathbf{n}=\mathbf{1 0 1 9})\end{array}$ & $\begin{array}{c}\text { Deceased } \\
(\mathbf{n}=\mathbf{1 3 9})\end{array}$ & $\begin{array}{c}\text { Survived } \\
(\mathbf{n}=\mathbf{3 8 5})\end{array}$ \\
\hline Age, median (range) - year & $70(65-81)^{* *}$ & $47(35-57)$ & $57(45-69)^{\diamond \diamond}$ & $32(24-44)$ \\
\hline Male - n (\%) & $26(70.3)^{*}$ & $510(50.0)$ & $74(53.2)^{\diamond}$ & $163(42.3)$ \\
\hline Comorbidities - \% & $24(64.9)$ & & $79(56.8)^{\diamond \diamond}$ & $69(17.9)$ \\
\hline Hypertension - \% & $18(48.6)$ & & $64(46.0)^{\diamond \diamond}$ & $44(11.4)$ \\
\hline Diabetes history - \% & $11(29.7)$ & & $30(21.6)^{\diamond \diamond}$ & $15(3.9)$ \\
\hline Cardiovascular disease - \% & $8(21.6)$ & & $40(28.8)^{\diamond \diamond}$ & $23(6.0)$ \\
\hline Chronic lung disease - \% & $3(8.1)$ & & $5(3.6)$ & $6(1.6)$ \\
\hline From onset to death, median (range) - day & $13(11-18)$ & & $15(10-19)$ & \\
\hline
\end{tabular}

Data are presented as medians (interquartile ranges, IQR) and $\mathrm{n}(\%)$.

${ }^{*} \mathrm{p}<0.05, * * \mathrm{p}<0.01$, vs. COVID-19 survived patients.

${ }_{\mathrm{p}}<0.05,{ }_{\mathrm{p}}<0.01$, vs. SARS-CoV survived patients.

deaths in Wuhan, 1019 recovered patients from six Chinese cities, and 524 SARS-CoV patients hospitalized in Beijing from March 25, 2003 to May 22, 2003 that included 139 deaths. ${ }^{26}$ Based on chi square analysis, the more serious cases involved men. In the COVID-19 deceased cohort, $70.3 \%$ were male; in the recovered cohort only $50 \%$ were male (Table 3 ). Similarly, $53.2 \%$ of the SARS-CoV deceased cohort were male, and $42.3 \%$ of the survivors were male (Table 3 ) ${ }^{26}$

In addition, this case series demonstrated nearly equal susceptibility between sexes with $50 \%$ patients among the 1019 recovered cohort being male and $51.2 \%$ among the 43 hospitalized patients. The SARS-CoV cohort also proved similar to the COVID-19 patients in that a higher proportion of males was among the deceased group along with having a higher mortality rate. A limitation of the study was that mortality and severity analysis were performed on the cohorts $<50$ due to unavailable public data. Together, these results demonstrate that the male gender appears to have a higher risk factor for increased severity and mortality in SARS and COVID-19 infections independent of age.

Hormonal and immune related differences between the sexes are likely components in the severity presentation and outcomes of SARS-CoV-2 patients. From a molecular standpoint, a key protein called angiotensin-converting enzyme 2 (ACE2) is a known component of COVID-19 pathogenesis. Perhaps coincidentally, the gene for ACE2 maps to the $X$ chromosome,${ }^{27}$ which also contains immune-related genes that are likely important in the effect of sex in viral infections. ${ }^{18}$

\section{THE ROLE OF ACE2 AND GENDER DIFFERENCES}

All three SARS-CoV, MERS-CoV, and COVID-19 pathogens share a similar mechanism of entry into the host by using spike protein interaction with receptors on host cell membranes. While MERS-CoV interacts with a DPP4 (dipeptidyl peptidase) receptor, ${ }^{28}$ both SARS-CoV and SARS-CoV-2 mediate entry by viral spike protein interaction with ACE2 receptors and the transmembrane protease serine 2 (TMPRSS2). ${ }^{29}$ Physiologically, ACE2 is a part of the renin-angiotensinaldosterone system (RAAS) that maintains blood pressure in humans (Figure 3). ${ }^{30}$ Specifically, ACE converts angiotensin I into angiotensin II (ATII), which then binds the angiotensin receptor, stimulating an increase in sympathetic nervous system tone, causing vasoconstriction and increasing blood pressure. ACE2 serves 


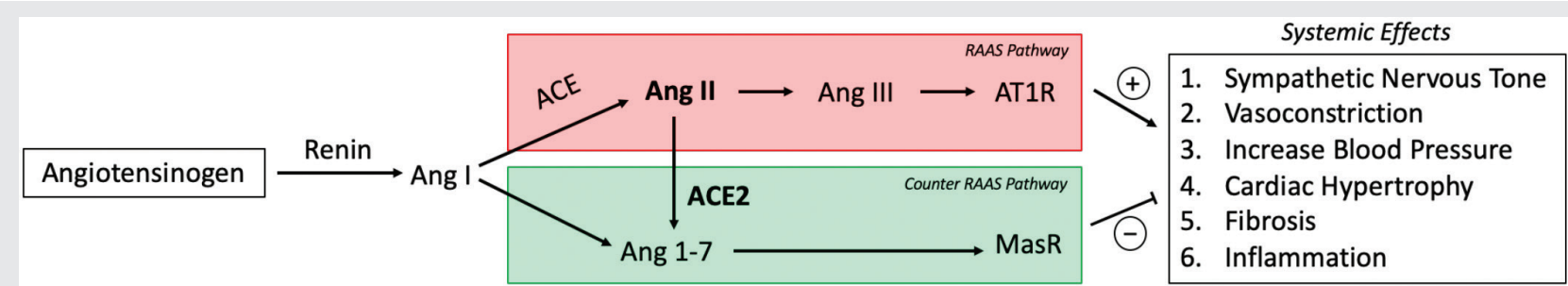

Figure 3. RAAS pathway adapted from Cheng et al. ${ }^{30}$

to breakdown/neutralize ATII by converting it to Ang 1-7. Subsequently, Ang 1-7 then binds to its receptor (MasR) and opposes the effects of ATII. As such, ACE2 can serve as a protective function in hypertension (HTN) and cardiovascular disease, by balancing the effects of ATII.

Furthermore, ACE2 is a membrane bound protein that is largely distributed in the intestine, heart, kidney, testis, and type II alveolar cells of the lung. ${ }^{30}$ The large surface area of alveolar tissue may explain the predilection of SARS-CoV-2 to infect pulmonary tissue. The binding of COVID-19 to ACE2 decreases the quantity of ACE2 available to degrade ATII, resulting in unopposed RAAS activity. COVID-19 infected patients with underlying causes of low ACE2 activity, such as hypertension, older age, diabetes, and cardiovascular disease, seem to be more at risk. ${ }^{27}$ With decreased ACE2 available one would think this could confer protection, yet SARS-CoV-2 binds ACE2 with a very high affinity. The ATII binding to its receptor is a potent promoter of inflammation in the lung that could be a factor in developing ARDS when infected with COVID-19. ${ }^{31}$ This inflammatory response is mediated by ATII binding that activates cytokine pathways, like TNF- $\alpha$ and NF- $\kappa B$, and releases IL-1 and IL- $6 .{ }^{31}$ At the forefront of treatment for COVID-19 infection are inhibitors of the RAAS system including ACE inhibitors, anti-IL-6 drugs, and AT1R inhibitors to reduce the dysregulated inflammatory response that contributes to ARDS. Yet due to uncertain results and conflicting information regarding these therapies, there is no definitive recommended treatment targeting ACE2. ${ }^{32}$

Differences have been shown in ACE2 expression between gender. In murine experiments conducted by Gupte et al., a high fat diet decreased renal ACE2 activity and increased blood pressure in males, while females had no decrease in adipocyte ACE2 activity and remained normotensive. After removing the ovaries in female mice, their ACE2 activity decreased and they became more obese and hypertensive. No other sex hormones besides estrogen increased expression of ACE2 mRNA in adipocytes. ${ }^{33}$ In fact, the loss of estrogen in post-menopausal women has shown to decrease cardiovascular protection due to overstimulation of the ACE/ATII/RAAS pathway. ${ }^{34}$

Xie and colleagues used a rat model to investigate differences in the expression of ACE2 in the lung in animals with different gender and age to study the pathogenesis of SARS-CoV and possible therapeutic treatment. ${ }^{35}$ The rats were divided into three age groups: young adult ( 3 months), middle-aged (12 months), and old (24 months). After the rats were sacrificed, their lungs were removed and frozen, and their respective ACE2 expression was determined using immunohistochemistry and Western blotting. ${ }^{35}$

Western blot results showed that ACE2 expression was lower in the old age group compared to the young adult group: $78 \%$ decrease in males and $67 \%$ decrease in females. The older group ACE2 expression was also decreased when compared to the middleaged group: $71 \%$ decrease in males and $59 \%$ decrease in females. However, the decrease from the young to middle age group was much less with a $25 \%$ reduction in males and an $18 \%$ reduction in females. Immunohistochemistry also demonstrated this phenomenon. ACE2 expression was not very different in male and female mice in the young and middle age group; higher ACE2 expression was found in females compared to males in the old group. These results suggest a possible reason for the more frequent viral 
infections in younger age individuals aged 20-39 in the 2003 SARS-CoV outbreak in China, since ACE2 serves as the entry receptor. ${ }^{35}$ Presented data also revealed that there was a greater female to male infection ratio (1.25:1), which contrasts the inverse ratio seen in both MERS-CoV (3.3:1) and SARS-CoV-2 (2.7:1). ${ }^{10}$

Increased ACE2 expression among young females correlated with increased infection of SARSCoV when compared to males, while COVID-19 has mostly occurred in the opposite group: elderly males. There must be other factors in COVID-19 pathogenesis that contribute to the increased severity and mortality in the older male age group. ${ }^{32}$

\section{RACIAL AND ETHNIC DISPARITIES IN RESPIRATORY VIRUSES}

Race is a categorical organization of people based on shared genetic and biological traits. When most people think about race, physical characteristics, such as hair texture, eye color, facial features, and skin tone come to mind. Ethnicity is defined as people who share the same nationality or cultural beliefs. An example of race would be Asian, while ethnicity would be Han Chinese. One can belong to multiple ethnic groups and be monoracial or multiracial. People of different ethnic backgrounds are vastly different in their ways of life, ranging from social behaviors to habits/ diets and health risks. ${ }^{36}$ This predisposes certain ethnic groups and races to poor disease outcomes in the setting of underlying comorbidity prevalence. For example, a study conducted by Tillin et al. showed both an increase in diabetes, coronary heart disease incidence, and stroke episodes among South Asian and African Caribbean people compared to Europeans. ${ }^{37}$

A current ongoing study by Cohall et al. seeks to determine if dysregulation between the ACE/ATII/AT1R and ACE2/Ang 1-7/MasR axes puts those of African descent at higher risk for hypertension. Reduced ACE2 has been seen in patients with prehypertension, diabetes mellitus type II, and chronic kidney disease. Hypertension is very common in patients of African descent and has a higher incidence of adverse outcomes, such as renal failure and stroke. Many genetic variants, such as CYP11B2 and SCNN1B, lead to renal pathologies such as primary aldosteronism and
Liddle Syndrome that contribute to dysfunction of the RAAS. Hypertensive African-Americans have some of these genes. ${ }^{38}$

With both the SARS-CoV and MERS-CoV outbreaks, most cases occurred in the region in which it began, i.e., Asia and the Middle East, respectively. Both of these viruses spread globally, but the total caseload for each virus were under $10,000 .^{10}$ Yet the global spread of COVID-19 infection is much larger, with over 3 million cases worldwide thus far. The COVID-19 virus has not affected all ethnicities and races in the same proportion. As of May 7, 2020, the United States has well over 1 million cases and 62,698 deaths, the highest in the world. ${ }^{39}$

The epicenter of the initial outbreak in the U.S. was in New York City, with the first case reported on March 1, 2020. ${ }^{40}$ As of May 4, 2020, New York City alone has reported 170,534 cases and over 13,000 deaths, with elderly males being the most affected. ${ }^{41}$ In New York City, African-Americans and other minority communities have been disproportionately affected by COVID-19. As of April 16, 2020, according to the New York City Health Department, for every 100,000 cases there have been the following rates of non-hospitalized patients: 333.5 African-American, 271.6 Hispanic, 190.4 White, and 95.1 Asian. In addition, 92.3 African-Americans and 74.3 Hispanics died per 100,000 as compared to 45.2 Whites and 34.5 Asians who died (Figure 4). Of those who were known to have died, $33.2 \%$ were African-Americans, $28.2 \%$ were Hispanics, and $30 \%$ were Whites. ${ }^{42}$

This trend does not restrict itself only to New York City. In Milwaukee, three quarters of all deaths due to COVID-19 were African-Americans, and in St. Louis, Missouri, as of April 14, 2020, all but three deaths were African-Americans. ${ }^{40}$ In San Diego as of May 5, 2020 , there have been 4,160 cases, with $55.2 \%$ of affecting the Hispanic/Latino population, 29\% Whites, and $4.5 \%$ African-American. ${ }^{43}$ Overall, U.S. reports have shown that in counties where black majority resides, the rate of COVID-19 infection is three times higher and the death rates are almost six times higher than in white majority counties. ${ }^{44}$ Native American populations also have a high risk of complications from COVID-19 due to many underlying conditions, such as heart disease 


\section{Age-adjusted Case Rate Per 100,000}
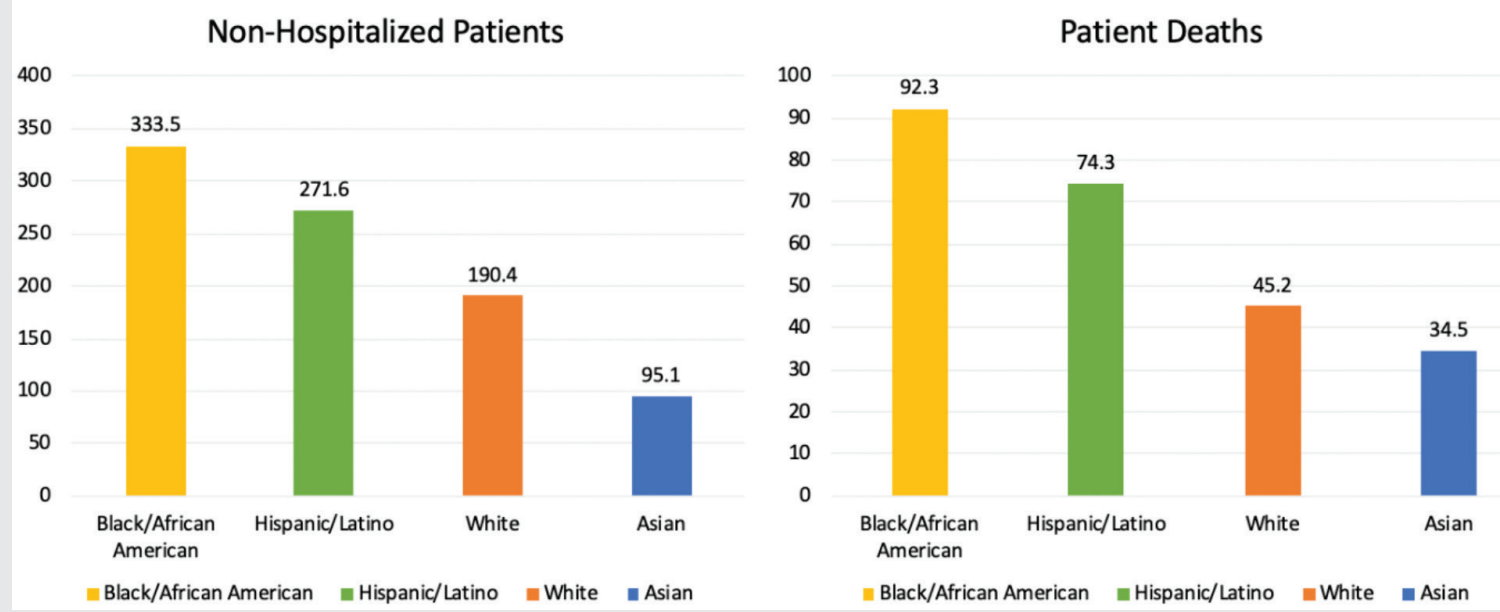

Figure 4. Data adapted from the New York City Health Department. ${ }^{41}$

and diabetes. Health care for Native Americans is provided under the treaty of obligations between the US government and the Indian Health Service (IHS). The IHS has limited resources for healthcare, and many Native Americans live long distances from IHS medical care..$^{40}$ In the United Kingdom, ethnic minorities make up $13 \%$ of England's population and about a third of the admissions to critical care due to COVID-19 infection. ${ }^{44}$ Out of 2249 patients admitted to the critical care units in England, $64.8 \%$ were white, $13.8 \%$ were Asian, $13.6 \%$ were Black, and $7.8 \%$ were of mixed ethnic backgrounds. Therefore, there is an urgent need to consider ethnicity and race when evaluating how to reduce the spread of COVID-19. ${ }^{44}$

Racial and ethnic minorities tend to live in densely populated underserved areas with residential segregation far from medical facilities that they would need most during disease outbreaks. ${ }^{16}$ The households of minorities may also be multigenerational, thus increasing the number of people in close proximity under one roof. Further close contact risks also include the disproportionately high incarceration rate among minorities, with the U.S. prison system having 2066 COVID-19 positive inmates as of May 5, 2020.45

Ethnic minorities in the United States are overwhelmingly employed in essential jobs where social distancing is not possible, such as grocery workers, custodial staff, and meat production plants. ${ }^{40}$ Furthermore, their jobs may not provide paid sick leave, thus forcing them to work even when they feel symptomatic. Compared to Caucasians, both Hispanics and African-Americans are less likely to have health insurance (Hispanics - 3x, African-Americans - 2x) and are also more likely to have underlying medical conditions and suppressed immune systems. ${ }^{16}$ Minority populations, especially African-Americans, have historically been disproportionately affected by diseases, such as HIV. Another population affected by disease and seldom considered in our modern age is indigenous inhabitants. These people tend to live in remote areas of the continent, without access to modern medicine. In South America, past epidemics have resulted in high mortality among indigenous people, and COVID-19 could be the next pathogen to cause high mortality rates. With these statistics and evident health disparities, it should not come as a surprise that ethnic minorities are being affected in a disproportionate manner. ${ }^{46}$

Many African-Americans live in tight knit communities within large cities, such as Detroit, Michigan, where infectious spread can be fast and potent. In Connecticut, the African-American population makes 
up only $4.9 \%$ of the population and at least one-third live in a few Connecticut cities. Compounded with limited health care access, higher poverty rates, and employment in jobs that cannot be performed from home, African-Americans are at greater risk for contracting COVID-19. In a study of 1528 diagnosed infectious patients, $55 \%$ of the cases did not have racial and ethnic data. Currently, there is no national United States registry information on race and ethnicity of COVID-19 cases. Missing data make investigating ethnic differences in the susceptibility of the virus very difficult to discern. ${ }^{47}$

\section{RACIAL AND ETHNIC BIOLOGICAL FACTORS AND SARS-CoV-2}

Both ACE2 and TMPRSS2 are expressed differently in various populations and are affected by comorbidities, such as diabetes mellitus. Researchers believe that ACE2 and TMPRSS2 are found in similar cells due to their correlated gene expression. Peters et al. analyzed sputum from asthmatic patients for ACE2 and TMPRSS2 gene expression to try to explain possible groups at risk for COVID-19 infection. From a 3-year longitudinal study using the Severe Asthma Research Program Protocol, 330 participants with asthma and 79 healthy patients were included. Sputum was collected from the patients after inhaling nebulized 3\% saline; then RNA was extracted and sequenced. ${ }^{48}$

There was no appreciable difference in the expression of ACE2 and TMPRSS2 between the healthy and asthmatic groups of patients. ACE2 and TMPRSS2 levels increased slightly with age, and both were significantly higher in males than in females. AfricanAmericans have known risk factors for hypertension due to polymorphisms in their renin-angiotensin pathway. The results in this study revealed that African-Americans had higher amounts of ACE2 and TMPRSS2 expression than Caucasians. When compared to Caucasians, Asian patients $(p=0.15)$ had 0.69 amount of TMPRSS2 while African-American patients had 1.28 times the amount of TMPRSS2 (significant with a $p<.001$ ). Expression of ACE2 was also higher in diabetics than in healthy patients, but there were no significant differences in expression between healthy and hypertensive patients. A few limitations of this study included mixed multiple cell types in the sputum samples, external data were not from a randomized placebo-controlled study, mRNA expressions do not always reflect protein levels, and conclusions were drawn on the larger cohort of asthmatic (330) rather than on healthy (79) patients. With these results, demographics with higher ACE2 expression, in particular African-Americans, would provide an additional rationale for studying the racial disparities in COVID-19 infection. ${ }^{48}$

Both ACE2 and TMPRSS2 are regulated by androgen receptors (AR). There is a 15 base pair promoter in the androgen response element for TMPRSS2, and it is the only known catalyst for this protease. There are wide genetic variations in androgen receptors across different ethnicities. African-Americans, for example, are more likely to have aggressive prostate cancer and exhibit alopecia due to polymorphisms in the exon 1 of their androgen receptors. The likelihood of prostate cancer can increase with decreasing CAG repeat length, with African-Americans having fewer CAG (19.8) repeats than white males (21.9). In males, the action of testosterone is dependent on how long the CAG repeat of the AR gene is. There have not been conclusive data on the correlation between CAG repeat lengths and the AR gene, but it is an important area of study. McCoy and colleagues have proposed a study to correlate CAG length polymorphisms in the AR COVID19 outcomes with respect to racial and gender biases. This could potentially bring anti-androgen therapies for consideration as a possible treatment for COVID-19 infection. ${ }^{49}$

\section{CULTURE DIFFERENCES AND TRANSMISSION OF VIRUSES}

Cultural roles and traditions have a significant effect on how certain groups go about their daily lives. Across the world, populations and communities consider certain roles among gender, age, and ethnicity/ race as normal, but they could be considered abnormal in other regions of the globe. All three COVID-19, SARS-CoV, and MERS-CoV infections have affected men more severely, due to known biological factors, such as comorbidities. Yet other factors not tied to biology, but to culture, could also be key in understanding 
viral transmission. A literature review conducted from the Department of Anthropology at the University of Toronto challenged the idea that the higher male infection rates of MERS-CoV were only due to biological phenomena, and demonstrated that they were also linked to gender roles, social constructs, and culture within Saudi Arabia. ${ }^{50}$

The author explored multiple Saudi Arabian social norms, including the religious Hajj, shisha smoking practices, gender roles of women, and livestock management of dromedary camels, the known intermediate host for MERS-CoV. Particularly in Saudi Arabia, women wear both a purdah and veil, covering both the nose and mouth. However, studies have shown that the microenvironment created in the facial area, the absence of a filterable material, loosefitting nature of the veil, and removal of facial coverings when at home could all leave females at risk for infection. ${ }^{50}$

Popular cafes in Saudi Arabia offer what is known as shisha, commonly referred to as hookah smoking, with most participants Middle Eastern men. As a result, within these cafes, there tends to be secondhand smoke, close contact with others, and sharing saliva through smoking mouthpieces, all of which could promote viral transmission. Both males and females participate in Haij and wear what is known as ihram which is standard dress that for men covers their body sparing the feet, hands, and face. For females, ihram still includes modest clothing that covers most of their body except the face. Therefore, men may be more at risk due to direct close contact, as more of their skin is exposed. ${ }^{50}$

MERS-CoV is known to have transmitted from bats to dromedary camels to humans (Figure 1). The dromedary camels in Saudi Arabia carry MERS$\mathrm{CoV}$ at higher rates than in other regions. The management of these camels involves large herds and farms that are run primarily by males. In addition, sports like camel racing are male dominated. Thus, this could be another cultural factor that predisposes males to MERS-CoV. Taken collectively, this analysis demonstrates how paramount cultural norms are in the transmission of infectious disease, not only with respect to gender bias but also racial and ethnic bias.
Analysis of such factors in different areas of the world could improve control, prevention, and treatment of SARS-CoV-2. ${ }^{50}$

\section{Discussion}

Our review article explores current available literature to establish a possible basis for the demographic discrepancies seen in the COVID-19 pandemic in comparison to other coronavirus outbreaks of the past. The most current COVID-19 statistical data were collected from respected agencies, including the World Health Organization and Centers for Disease Control and Prevention.

There exists a sex bias in mortality in COVID-19 infected patients, in which males are more likely to have poor outcomes. ${ }^{12}$ Multiple studies were reviewed that analyzed available data from COVID-19, MERSCoV, and SARS-CoV infections and supported this gender difference. The complexity underlying the biological differences between the sexes can make effectively addressing this bias difficult. The $X$ chromosome contains immune related genes, and females tend to have more robust immune responses to infectious agents than males. Male COVID-19 patients have less IgG plasma antibody production in severe cases. ${ }^{20}$ Understanding these differences in immune responses during active infection could provide a blueprint from which to not only predict outcomes but perhaps stimulate future immunotherapy development. Males and females also differ in their sex steroid production and receptor density on immune cells. Estrogen has been shown to be protective and decrease the inflammatory response in lung tissue in ARDS. ${ }^{23}$ In addition, ACE2 serves as the receptor for the viral entry of COVID-19 and SARS-CoV; it also opposes the RAAS that when uncontrolled leads to inflammatory effects. Based on animal studies, ACE2 expression has been shown to be lower in elderly samples and higher in females than males. This explains the higher female to male infection ratio in the SARS-CoV outbreak, yet males still made up the larger percentage of deaths. In contrast, elderly males are most affected by COVID-19. As such, the mechanistic effects of ACE2 levels and other possible 
pathophysiological processes must be investigated further to explain these differences. ${ }^{33,35}$

There are limited international data on COVID-19 among racial and ethnic groups; epidemiological studies in the United States have shown that AfricanAmericans are disproportionately more affected than other groups. Although they make up a small percentage of the U.S. population, they have been infected and died from COVID-19 at higher rates. ${ }^{14,41}$ Many ethnic groups are naturally at higher risk for contracting respiratory viruses due to inadequate living conditions, poor healthcare access, occupational exposure, and cultural norms. ${ }^{16,40,50}$ These factors can be applied to various races and ethnic groups living in other areas of the world with indigenous peoples. Thus, the societies in which these vulnerable groups live must be kept accountable in reducing the spread of COVID-19. In addition, there are biological differences between people throughout the world that have evolved over time that may predispose them to illness. AfricanAmericans have been shown to be prone to hypertension, which correlates with lower ACE2 expression. ${ }^{38}$ Yet they have also been shown to have higher ACE2 and TMPRSS2 expression than Whites, perhaps providing a basis for their poor outcomes. Regardless, the small sample size and contradicting findings do not provide a clear biological mechanism for their increased risk. ${ }^{48}$

This review has several limitations. Results of the studies with a small sample size should be considered based on context and how they could apply to future, larger studies. Excluding literature in languages other than English could result in missing studies that support or contradict certain findings. Last, selection of the mentioned literature could be complicated by unknown bias.

The demographic differences in COVID-19 infection with respect to gender, sex, race, and ethnicity are multifactorial and complex. They involve biological and societal components that should be pursued separately to clarify plausible explanations for potential future solutions and for possible preventative measures to limit pandemics such as the COVID-19 viral infection.
Article citation: Ha FL, John A, Zumwalt M. Gender/ sex, race/ethnicity, similarities/differences among SARS-CoV, MERS-CoV, and COVID-19 patients. The Southwest Respiratory and Critical Care Chronicles 2021;9(37):9-23

From: The School of Medicine (FLH, AJ) and the Department of Orthopedics (MZ), Texas Tech University Health Sciences Center, Lubbock, Texas

Submitted: $5 / 16 / 2020$

Accepted: $12 / 24 / 2020$

Reviewer: Kenneth Nugent MD

Conflicts of interest: none

This work is licensed under a Creative Commons

Attribution-ShareAlike 4.0 International License.

\section{REFERENCES}

1. Zhou P, Yang X-L, Wang X-G, et al. A pneumonia outbreak associated with a new coronavirus of probable bat origin. Nature [Internet] 2020;579(7798):270-3.

2. Fu L, Wang B, Yuan T, et al. Clinical characteristics of coronavirus disease 2019 (COVID-19) in China: A systematic review and meta-analysis. J Infect [Internet]. 2020 Apr 10; S0163-4453(20)30170-5.

3. WorldHealthOrganization.WHOTimeline-COVID-19[Internet]. [cited 2020 May 4]. Available from: https://www.who. int/news-room/detail/27-04-2020-who-timeline-covid-19

4. World Health Organization. WHO COVID-19 Dashboard [Internet]. [cited 2020 May 6]. Available from: https://covid19. who.int/

5. Andersen KG, Rambaut A, Lipkin WI, et al. The proximal origin of SARS-CoV-2. Nat Med [Internet]. 2020;26(4):450-2.

6. Rabi AF, Al Zoubi SM, Kasasbeh AG, et al. SARS-CoV-2 and Coronavirus Disease 2019: what we know so far. Vol. 9, Pathogens 2020.

7. Zhang T, Wu Q, Zhang Z. Probable pangolin origin of SARSCoV-2 associated with the COVID-19 Outbreak. Curr Biol [Internet]. 2020;30(7):1346-1351.e2.

8. Centers for Disease Control and Prevention. SARS Basic Fact Sheet [Internet]. CDC. 2004. Available from: https:// www.cdc.gov/sars/surveillance/absence.pdf

9. Centers for Disease Control and Prevention. Information about Middle East Respiratory Disease (MERS) [Internet]. 2015. p. 1-2. Available from: www.cdc.gov/coronavirus/mers

10. Wang C, Horby PW, Hayden FG, et al. A novel coronavirus outbreak of global health concern. Lancet (London, England) [Internet]. 2020/01/24. 2020 Feb 15;395(10223):470-3. 
11. Johns Hopkins University. Mortality Analyses - Johns Hopkins Coronavirus Resource Center [Internet]. [cited 2020 May 6]. Available from: https://coronavirus.jhu.edu/data/mortality

12. Global Health 5050. COVID-19 sex-disaggregated data tracker [Internet]. [cited 2020 May 6]. Available from: https://globalhealth5050.org/covid19/\#1586248980572-383 9d9fe-3b88

13. World Health Organization Europe. COVID-19 weekly surveillance report [Internet]. [cited 2020 May 6]. Available from: http://www.euro.who.int/en/health-topics/healthemergencies/coronavirus-covid-19/weekly-surveillancereport

14. Centers for Disease Control and Prevention. COVID-19 Laboratory-Confirmed Hospitalizations [Internet]. [cited 2020 May 6]. Available from: https:/gis.cdc.gov/grasp/COVIDNet/ COVID19_5.html

15. U.S. Census Bureau. U.S. Census Bureau QuickFacts: United States [Internet]. [cited 2020 May 6]. Available from: https:// www.census.gov/quickfacts/fact/table/US/PST045219

16. Centers for Disease Control and Prevention. COVID-19 in Racial and Ethnic Minority Groups | CDC [Internet]. [cited 2020 May 5]. Available from: https://www.cdc.gov/coronavirus/ 2019-ncov/need-extra-precautions/racial-ethnic-minorities. html

17. Klein SL, Flanagan KL. Sex differences in immune responses. Nat Rev Immunol [Internet]. 2016;16(10):626-38.

18. Schurz H, Salie M, Tromp G, et al. The X chromosome and sex-specific effects in infectious disease susceptibility. Hum Genomics. 2019;13(1):2.

19. Klein SL. Sex influences immune responses to viruses, and efficacy of prophylaxis and treatments for viral diseases. Bioessays [Internet]. 2012/09/26. 2012 Dec;34(12):1050-9.

20. Zeng F, Dai C, Cai P, et al. A comparison study of SARS-CoV-2 IgG antibody between male and female COVID-19 patients: a possible reason underlying different outcome between gender. medRxiv [Internet]. 2020 Jan 1;2020.03.26.20040709. Available from: http://medrxiv.org/content/early/2020/03/27/ 2020.03.26.20040709.abstract

21. Kadel S, Kovats S. Sex hormones regulate innate immune cells and promote sex differences in respiratory virus infection. Front Immunol. 2018;9(JUL):1-15.

22. Jaillon S, Berthenet $\mathrm{K}$, Garlanda C. Sexual Dimorphism in Innate Immunity. Clin Rev Allergy Immunol. 2019;56(3): 308-21.

23. Channappanavar R, Fett C, Mack M, et al. Sex-Based Differences in Susceptibility to Severe Acute Respiratory Syndrome Coronavirus Infection. J Immunol. 2017;198(10):4046-53.

24. Karlberg J, Chong DSY, Lai WYY. Do men have a higher case fatality rate of severe acute respiratory syndrome than women do? Am J Epidemiol [Internet]. 2004 Feb 1;159(3): 229-31.
25. Jansen A, Chiew M, Konings F, et al. Sex matters - a preliminary analysis of Middle East respiratory syndrome in the Republic of Korea, 2015. West Pacific Surveill response J WPSAR [Internet]. $2015 \mathrm{Jul}$ 22;6(3):68-71.

26. Jin J-M, Bai P, He W, et al. Gender differences in patients with COVID-19: Focus on severity and mortality. medRxiv [Internet]. 2020 Jan 1;2020.02.23.20026864. Available from: http:// medrxiv.org/content/early/2020/03/05/2020.02.23.20026864. abstract

27. Verdecchia P, Cavallini C, Spanevello A, et al. The pivotal link between ACE2 deficiency and SARS-CoV-2 infection. Eur J Intern Med [Internet]. 2020 Apr 20; Available from: https://pubmed.ncbi.nlm.nih.gov/31706707

28. Wan Y, Shang J, Sun S, et al. Molecular mechanism for antibody-dependent enhancement of coronavirus entry. Gallagher T, editor. J Virol [Internet]. 2020 Feb 14;94(5):e02015-19. Available from: http://jvi.asm.org/content/94/5/e02015-19. abstract

29. Hoffmann M, Kleine-Weber H, Schroeder S, et al. SARSCoV-2 cell entry depends on ACE2 and TMPRSS2 and is blocked by a clinically proven protease inhibitor. Cell [Internet]. 2020;181(2):271-280.e8. Available from: http://www. sciencedirect.com/science/article/pii/S0092867420302294

30. Cheng H, Wang Y, Wang G-Q. Organ-protective effect of angiotensin-converting enzyme 2 and its effect on the prognosis of COVID-19. J Med Virol. 2020;(March):1-5.

31. HiranoT, Murakami M. COVID-19: A new virus, but a familiar receptor and cytokine release syndrome. Immunity [Internet]. 2020;0(0):3-5. Available from: https://linkinghub.elsevier. com/retrieve/pii/S1074761320301618

32. Ardes DD, Boccatonda A, Rossi I, et al. COVID-19 and RAS: unravelling an unclear relationship. 2020;2:1-8.

33. Gupte M, Thatcher SE, Boustany-Kari CM, et al. Angiotensin converting enzyme 2 contributes to sex differences in the development of obesity hypertension in C57BL/6 mice. Arterioscler Thromb Vasc Biol. 2012;32(6):1392-9.

34. Bukowska A, Spiller L, Wolke C, et al. Protective regulation of the ACE2/ACE gene expression by estrogen in human atrial tissue from elderly men. Exp Biol Med (Maywood) [Internet]. 2017/06/29. 2017 Aug;242(14):1412-23.

35. Xie X, Chen J, Wang X, et al. Age- and gender-related difference of ACE2 expression in rat lung. Life Sci [Internet]. 2005/11/21. 2006 Apr 4;78(19):2166-71.

36. Pareek M, Bangash MN, Pareek N, et al. Ethnicity and COVID-19 : an urgent public health research priority. Lancet [Internet]. 2020;6736(20):2019-20.

37. Tillin T, Hughes AD, Mayet J, et al. The relationship between metabolic risk factors and incident cardiovascular disease in Europeans, South Asians, and African Caribbeans: SABRE (Southall and Brent Revisited) - A prospective populationbased study. J Am Coll Cardiol. 2013;61(17):1777-86. 
38. Cohall D, Ojeh N, Ferrario CM, et al. Is hypertension in African-descent populations contributed to by an imbalance in the activities of the ACE2/Ang-(1-7)/Mas and the ACE/Ang II/AT(1) axes? J Renin Angiotensin Aldosterone Syst [Internet]. 2020;21(1):1470320320908186-1470320320908186. Available from: https://pubmed.ncbi.nlm.nih.gov/32089050

39. World Health Organization. United States of America: WHO COVID-19 Dashboard [Internet]. [cited 2020 May 7]. Available from: https://covid19.who.int/region/amro/country/us

40. Dorn A van, Cooney RE, Sabin ML. COVID-19 exacerbating inequalities in the US. Lancet (London, England) [Internet]. 2020 Apr 18;395(10232):1243-4.

41. New York City Health Department. COVID-19: Data-NYC Health [Internet]. [cited 2020 May 5]. Available from: https://www1.nyc.gov/site/doh/covid/covid-19-data.page

42. NYC Health. Age-adjusted rates of lab confirmed COVID-19 non hospitalized cases,estimated non-fatal hospitalized cases, and patients known to have died 100,000 by race/ethnicity group as of April 16, 2020 [Internet]. [cited 2020 May 5]. Available from: https://www1.nyc.gov/assets/doh/downloads/ pdf/imm/covid-19-deaths-race-ethnicity-04162020-1.pdf

43. County of San Diego Human and Health Services Agency. County of San Diego Daily 2019 Novel Coronavirus (COVID19) Race/Ethnicity Summary [Internet]. [cited 2020 May 5]. Available from: https://www.sandiegocounty.gov/content/dam/ sdc/hhsa/programs/phs/Epidemiology/COVID-19 Race and Ethnicity Summary.pdf
44. Khunti K, Singh AK, Pareek M, et al. Is ethnicity linked to incidence or outcomes of covid-19? BMJ [Internet]. 2020;369. Available from: https://www.bmj.com/content/369/ bmj.m1548

45. Federal Bureau of Prisons. BOP: COVID-19 Update [Internet]. [cited 2020 May 5]. Available from: https://www.bop. gov/coronavirus/

46. Ferrante L, Fearnside PM. Protect indigenous peoples from COVID-19. Science(80-).2020Apr 17;368(6488):251.1-251.

47. Laurencin CT, McClinton A. The COVID-19 pandemic: a call to action to identify and address racial and ethnic disparities. J Racial Ethn Heal Disparities [Internet]. 2020; Available from: https://doi.org/10.1007/s40615-020-00756-0

48. Peters MC, Sajuthi S, Deford P, et al. COVID-19 Related genes in sputum cells in asthma: relationship to demographic features and corticosteroids. Am J Respir Crit Care Med [Internet]. 2020;rccm.202003-08210C. Available from: https:// www.atsjournals.org/doi/10.1164/rccm.202003-0821OC

49. McCoy J, Wambier CG, Vano Galvan S, et al. Racial variations in COVID 19 deaths may be due to androgen receptor genetic variants associated with prostate cancer and androgenetic alopecia. are anti androgens a potential treatment for COVID 19? J Cosmet Dermatol. 2020;

50. Ali MA. Gender dynamics and socio-cultural determinants of middle east respiratory syndrome coronavirus (MERSCov) in Saudi Arabia. Univ Toronto Med J. 2017;94(1): $32-7$. 